\title{
Process Analysis of Condition Based Maintenance for Equipment
}

\author{
Chunjian Wang ${ }^{1, ~ a ~}$, Hongjun Fan ${ }^{2, b}$ and Tiao Wang ${ }^{3, c}$ \\ ${ }^{1}$ Navy Submarine Academy, Qingdao, 266042, China \\ ${ }^{2}$ Qingdao Branch of Naval Aeronautical Engineering Institute, Qingdao, 266042, China \\ ${ }^{3}$ Navy Submarine Academy, Qingdao, 266042, China \\ a,b,czhihe_wcj@163.com
}

Keywords: CBM; Proactive maintenance; Process; state; Prediction

\begin{abstract}
For a long time, the instruction of navy equip.'s detections and maintenance is the traditional theories, and the plan servicing is the main instruction thought and organized form. But with the development of electrification and digitization, the traditional method may cause disrepair and excessive repair. It is hard to satisfy the maintenance support requirement of equipment, and restrict the formation and advance of fighting strength. The paper researches the characteristic of $\mathrm{CBM}$ and the choice of object and the choice of state information parameter and the choice of monitor means and etc., and provides a set of method and thinking to develop the maintenance of condition based maintenance for equipment.
\end{abstract}

\section{Introduction}

The theories of condition based maintenance and proactive maintenance get development in civil realm. But it is not enough in military realm. The main reason is how to build a bridge between the theories and equip manage, and it provides the equip manager with the method that how to develop scientific management. The paper researches the characteristic of CBM and the choice of object and the choice of state information parameter and the choice of monitor means and etc., and provides a set of method and thinking to develop the maintenance of condition based maintenance for equipment.

In order to realize the CBM, the first is to estimate the state of equip, and then makes the repairing method based on the state. In other words, it includes state detections and fault diagnosis and maintenance decision. The state detection is using the instrument to show the parameter, and fault diagnosis and estimation is analyzing the reason and place and degree based on the various parameters from state decision-making system. It analyzes and judges the operating conditions by state data, and provides data support for repair decision [1-2].

\section{State Detection}

State detection is the first step to carry out the maintenance decision based on the status, and is the precondition and base to forecast the residual life and optimize the maintenance decision. It can detect cerntain state parameter of object by monitoring, and valuate the operating conditions primarily. In the actual engineering application, two types of problems should be solved, and one is characteristic parameter that needs to be detected, and the other is the suitable detecting technique.

Confirm the state detecting characteristic parameter

During the operating, it can produce all kinds of physical phenomenon that represents the state, such as vibration and noise and temperature and etc... And it can cause the change of corresponding parameter. All these provide the possibillity for the state detection. In actual application, we should choose the parameters that reflect the operating state most to detect.

Choose the state detecting technique

After confirming the characteristic parameter of detecting equip, we shuold choose the suitable detecting technique to achieve the state deteccting information. Currently, there are many kinds of detecting technique that can be used in actual engineering, such as kinetic effect and chemical effect 
and physical effect and so on. Among them, kinetic effect and chemical effect are the most extensive technique currently.

\section{Process the State Information}

When detecting the state, it is necessary to use sensor and measure instrument to change the characteristic parameter into discrete or continuous electrical signal. These original electrical signals contain the important information of equip or assembly state. Two problems shuold be solved if these electrical signals can be used in CBD. One is how to eliminate the noise interference of original signals with maximum limit, and then give prominence to the useful information. The other is how to process and analyze the signal, and then find the inside regulation. An effective path to solve the problem is the processing of state information, and it is an important part in CBM decision. In the actual application, the process of signal treatment is as follows:

The pretreatment of signal

The signal acquiring by state detecting includes noise and information. The purpose of signal pretreatment is eliminating the influence of noise in the maximun limit and improving the signal-to-noise ratio and standing out the useful information.

Confirming the signal character

The signals acquiring from state detecting have many types and have its character respectively. It needs to describe them with different parameter and needs to use different methods to acquire the parameter. The familiar testing of signal character has periodic and random testing and ergodicity testing and stationarity testing.

The processing and analysis of signal

The work that process and analyze the signal is acrried on crosswise.

\section{Residual Life Prediction}

The residual life prediction is the key of making the CBM decision. In is necessary to predicte the residual life in order to realize the scientific and quantificational follow-up decision.

The definition of residual life is using certain predicting method to estimate the residual life based on the historical and current state. In this paper, the residual life does not limit in the specialized sense.

Choose the life characteristic parameter

Choosing the life characteristic parameter is the first step to carry on residual life prediction, and is the precondition and base of the following link. Usually, the parameter that can stand for the life characteristic has residual life and reliability and risk rate etc.

Establish or choose the model

After confirming the life characteristic parameter, how to describe the relationship between state information and life characteristic parameter becomes the key of the whole process. An effective path to solve the problem is establishing or choosing the mathematic model. We choose operating time and state information as variate, and describe the relationship accurately by mathematic expression. Generally speaking, it needs eatablish or choose different method because of different characteristic parameter. Now, the residual life prediction models that can be choosed and mature have filtering model and proportional model. They chooses residual life distribution density function and risk ratio as life characteristic parameter respectively, and estabish the model, and realize the the quantificational description between state information and life characteristic.

Parameter estimation and testing

It is ineluctable to induct some parameters during the process of choosing or establishing the model. These parameters reflect the whole life characteristic of equip from certain degree, and it is important for the whole residual life prediction. Therefore, we need to eatimate the parameters in model based on the state information data and life inforamtion data. In the actaul engineering application, we 
choose estimation method according to the data quantity, such as maximal likelihood estimation and least square estimation [3-4].

After eatimation, it is necessary to adopt certain model to test the estimated parameters whether it is acceptable. The familiar testing method includes likelihood ratio testing and $\mathrm{x}^{2}$ testing [5-6].

\section{Make Maintenance Decision}

Making maintenance decision is the last step of CBM, and it is the finally purpose. The main order is decided whether the equip needs respair, and if needs when carry on the respair, and reach the decision with minimum resources depletion. There are two aspects in making the mainteance decision, confirming the target and building and solving the target function.

Confirm the maintenance decision

Confirming the decision target is the first step to make maintenance decision. We can choose different target according to the actual need. For example, when the fault may induce fateful safety influence, such as personnel die or injure, or pollution of the environment, the decision should take safety as the target. When the fault influences the completion of task, should take task as the target. When the fault can not influence the safety and completion, we should think about the economic loss that induced by the fault, and should take economical efficiency as the target.

Establish and solve the decision target function

After confirming the decision target, it needs to estabilsh homologous target function. In the actual application, we can choose the suitable target function according to different decision target. For the safety target, we use fault rate or risk rate as target function. For the task target, we use average availablity as target function. For economy target, we use average expense in per unit time as target function. Then, we can get the optimal maintenance time under different decision target by numerical computation, and make the optimal maintenance decision under different constraint condition.

\section{Conclusions}

In one word, the process of CBM is as figure 1.during the state detecting stage, the main work is collecting and disposing the diagnosis signal, and distilling and disposing the eigenvalue, and judging whether the value overstep the threshold value. If it does not overstep, then return to signal collection. If it do overstep, then go to the next stage. During the fualt diagnosis and prediction stage, the main work is juding the faulat type. If it is latent fault, then predicts the residual life.if it is not, and then judges as functional fault. During the decision stage, we can decide whether mainteance is needed and when carry on the maintenance and how to maintain based on the state information and life prediction. That is to say, draw up a maintenance plan. The finally step is implementing the maintenance. It is carriying on preventive maintenance for the latent fault, and repairing maintenance for the functional fault. 


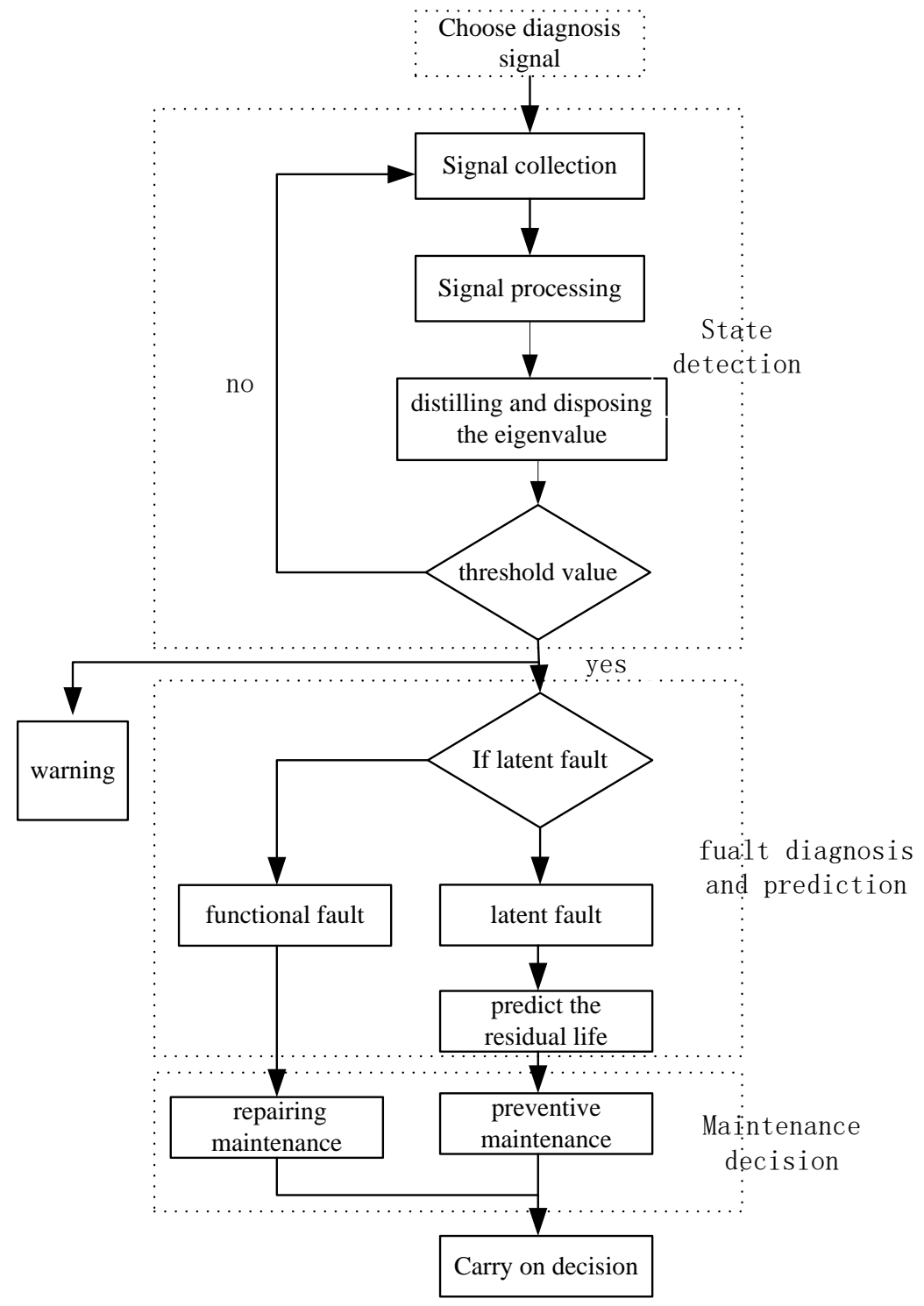

Fig.1 Process of condition based maintenance

\section{References}

[1] Wan Anping: CBM decision-making system based on real-time status monitoring for gas turbine. Electric Power Automation Equipment, 2013.33(7), p. 155-160.

[2] Yun Jian: New progress and future prospects of CBM exploration and development in China. Journal of China University of Mining \& Technology, 2012.22(3), p. 363-369.

[3] Zhou Zhi: Fault diagnosis of rolling bearings based on EMD Interval-Threshold denoising and maximum likelihood estimation. Journal of Vibration and Shock 2013.32(9) , p. 155-159.

[4] Liao Ying: Satellite precise orbit determination method based on penalized least squares estimation. Systems Engineering and Electronics, 2012, 34(7), p. 1430-1434.

[5] Hu Zhen-tao: Residual likelihood ratio test for fault diagnosis based on cost reference particle filter, SYSTEMS ENGINEERING AND ELECTRONICS, 2009, 31(12) , p. 3022-3025.

[6] Li Zheng-zhou: Infrared dim target track initiation method based on likelihood ratio test. High Power Laser and Particle Beams, 2012, 24(1), p. 75-78. 
[7]Zhao Jun-feng, Zhou Jian-tao, Xing Guan-nan: Research of Translating UML Activity Diagram to Petri Net. Computer Science, 2014, 41(7):143-147.

[8]Wang Yuhua, Yin Guisheng: Automatically generating a UML model from a requirement model based on ontology. Journal of Harbin Engineering University, 2012, Vol.33, No.12:735-740.

[9]CHAI Yu-mei,FENG Qiu-yan,WANG Li-ming: Research on Methods for Generating Test Cases of Inter-Classes Interaction Based on UML Models and OCL Constraints. Acta Electronica Sinica, 2013(6):1242-1248.

[10]Song A-ni, Wang Ming-zhe, Guo Fa-bin, Ni Feng: Unfolding the basic structure of colored Petri nets. SYSTEMS ENGINEERING - THEORY \& PRACTICE, 2011, Vol.31, No.2:316-322.

[11]Wu Guo-guang, Liu Gui-xiong, Zhou Song-bin: Modeling and Dynamic Performance Evaluation of WTIM Based on Petri Nets. Journal of South China University of Technology (Natural Science Edition), 2013, Vol.41, No.9:108-112. 